\title{
Intervenção fisioterapêutica na Síndrome da Imobilidade em pessoas idosas: revisão sistematizada
}

\author{
Physiotherapeutic intervention in Imobility Syndrome in \\ elderly people: a literature review \\ Intervención fisioterapéutica en el Síndrome de La Inmobilidad em personas \\ idosas: una revisión de literatura \\ Hellen Cristina Barbosa PEREIRA ${ }^{1}$ \\ Paulo Henrique Meira DUARTE ${ }^{1}$ \\ Thaynara do Monte MÉLO $^{1}$ \\ Rielly Maria Cruz da SILVA ${ }^{1}$ \\ Wagner Vitória dos SANTOS ${ }^{2}$ \\ Daniella de Souza BARBOSA \\ Rafaela Gerbasi NÓBREGA ${ }^{3}$ \\ Laura de Sousa Gomes VELOSO ${ }^{3}$ \\ ${ }^{I}$ Fisioterapeutas pela Faculdade Maurício de Nassau, UNINASSAU, \\ 58030-000 João Pessoa - PB, Brasil \\ ${ }^{2}$ Fisioterapeuta pela Faculdade de Ciências Médicas, 58010-740 João Pessoa-PB, Brasil \\ ${ }^{3}$ Docente Mestre do Curso de Fisioterapia da Faculdade Maurício de Nassau, UNINASSAU, \\ 58030-000 João Pessoa - PB, Brasil
}

\begin{abstract}
Resumo
O envelhecimento é um estado comum na qual todo idoso irá vivenciar, no entanto envelhecer não é apenas um estado, e sim um processo que o corpo viverá. Como consequência a saúde do idoso é marcada pelo desempenho proporcional de quatro esferas funcionais: cognição, humor, mobilidade e comunicação. Com isso o presente estudo tem por objetivo conhecer as atuais tendências da produção cientifica nacional e internacional sobre a intervenção fisioterapêutica na síndrome da imobilidade em pessoas idosas. Material e método: Para tal foi realizado estudo de revisão de literatura, fundamentada em livros e ensaios científicos publicados sobre a intervenção da fisioterapia na patologia supracitada. Foram analisados 7 estudos, sendo possível observar que a atuação fisioterapêutica na síndrome da imobilidade é extremamente importante, mostrando a eficácia nas respostas negativas que a patologia traz, tendo a cinesioterapia como recurso mais utilizado. Foi constatado ainda que ainda há muito que se investigar sobre essa temática.

Descritores: Idoso; Movimento; Síndrome; Fisioterapia.
\end{abstract}

\begin{abstract}
Aging is a common state in which every elderly person will experience, but aging is not just a state, but a process that the body will live. As a consequence the health of the elderly is marked by the proportional performance of four functional spheres: cognition, humor, mobility and communication. The objective of this study is to understand the current trends in the national and international scientific production on physiotherapeutic intervention in immobility syndrome in elderly people. Material and method: This study was carried out to review the literature, based on published books and scientific studies on the intervention of physiotherapy in the aforementioned pathology. Were analyzed 7 studies and it was possible to observe that the physiotherapeutic action in the immobility syndrome is extremely important, showing the efficacy in the negative responses that the pathology brings, with kinesiotherapy being the most used resource. It was also verified that there is still much to investigate on this subject.
\end{abstract}

Descriptors: Aged; Movement; Syndrome; Physical Therapy Specialty.

\section{Resumen}

El envejecimiento es un estado común en el que todos los ancianos vivencian, pero envejecer no es sólo un estado, sino un proceso que el cuerpo vivirá. Como consecuencia la salud del anciano está marcada por el desempeño proporcional de cuatro esferas funcionales: cognición, humor, movilidad y comunicación. Con esto el presente estudio tiene por objetivo conocer las actuales tendencias de la producción científica nacional e internacional sobre la intervención fisioterapéutica en el síndrome de la inmovilidad en personas ancianas. Material y método: Para ello se realizó un estudio de revisión de literatura, fundamentada en libros y ensayos científicos publicados sobre la intervención de la fisioterapia en la patología anteriormente citada. Fueron analizados 7 estudios, fue posible observar que la actuación fisioterapéutica en el síndrome de la inmovilidad es extremadamente importante, mostrando la eficacia en las respuestas negativas que la patología trae, teniendo la cinesioterapia como recurso más utilizado. Se constató que todavía hay mucho que investigar sobre esta temática.

Descriptores: Anciano; Movimiento; Síndrome; Fisioterapia.

\section{INTRODUÇÃO}

Fala-se comumente do envelhecimento como um estado em que todo indivíduo irá vivenciar e que se classifica como "terceira idade" ou até "quarta idade". Porém, envelhecer não é apenas um estado, mas sim um processo que o corpo experimenta a degeneração progressiva e diferencial. O modo através do qual o indivíduo pode envelhecer dependerá muito de três fatores: biológico, psicológico e sociológico, e varia muito de pessoa para pessoa ${ }^{1}$.

Assim, pode-se assegurar que os indivíduos envelhecem de formas muito distintas e, a este respeito, podemos nos referir à idade biológica, cronológica, idade social e psicológica. Dentro desses processos de envelhecimento pode-se encontrar a senescência e senilidade quando há modificações fisiológicas e somatório de diversos mecanismos patológicos respectivamente ${ }^{1-3}$. A saúde do idoso está intimamente ligada com sua funcionalidade geral, dita como capacidade de regular a própria vida ou cuidar de si.

O idoso é considerado saudável a partir do momento que conseguir realizar tudo com independência e autonomia, mesmo havendo presença de doença. Capacidade essa que é possível ser avaliada através da análise das atividades de vida diária (AVDs) ${ }^{4}$. E a 
independência diz respeito à capacidade do individuo de desempenhar atividades de forma própria, significa dizer que o individuo depende diretamente de mobilidade e comunicação. Consequentemente a saúde do idoso é marcada pelo desempenho proporcional de quatro esferas funcionais: cognição, humor, mobilidade e comunicação ${ }^{4}$.

A síndrome da imobilidade é um conjunto de modificações que o individuo sofre decorrente de um longo período acamado, independente das causas que o motivou a tal situação, esse conjunto de sinais e sintomas pode evoluir para um quadro de problemas circulatórios, dermatológicos, respiratórios e na maioria das vezes psicológicos.

Dessa forma pode-se dizer também que problemas de origem ortopédica e diversos tipos de infecções são desordens que trazem muita dor ao individuo, levando-o a permanecer por um longo tempo restrito ao leito. Alterações psiquiátricas também são fatores que levam o desenvolvimento da síndrome da imobilidade ${ }^{5}$.

O Fisioterapeuta irá trabalhar diretamente nas consequências da doença resultante da imobilização no leito, principalmente no sistema musculoesquelético, tais quais encurtamentos, diminuição da amplitude de movimento (ADM), diminuição da mobilidade e flexibilidade, além de aumento da tensão muscular, que muitas vezes se instala devido ao longo tempo na mesma posição, diminuição da força muscular, complicações pulmonares e edemas ${ }^{4,6}$.

Dentre as síndromes geriátricas a síndrome da imobilidade é a de maior taxa de morbimortalidade dentro do universo sindrômico, uma vez que $40 \%$ dos idosos que desenvolvem a síndrome vão a óbito, decorrente na maioria das vezes por falência de múltiplos órgãos ou por patologias especificas como: pneumonia, embolia pulmonar ou septicemia, sendo essas as mais comuns ${ }^{7}$. As condições clínicas dos pacientes com síndrome da imobilidade e o falta de capacitação dos profissionais da área de saúde que atuam junto à população geriátrica concorrem para esses índices.

Tendo em vista o grande índice de mortalidade dos idosos acometidos pela síndrome e o desconhecimento dos profissionais de saúde que lidam com os idosos, dentre eles fisioterapeutas, e a baixa produção científica sobre os efeitos da fisioterapia, houve o interesse em buscar na literatura atual, a atuação da fisioterapia na síndrome da imobilidade. Surgindo a seguinte problemática: Quais as atuais tendências na produção científica nacional e internacional sobre a intervenção fisioterapêutica na síndrome da imobilidade em pessoas idosas.

Portanto, o presente estudo tem por objetivo conhecer as atuais tendências da produção cientifica nacional e internacional sobre a intervenção fisioterapêutica na síndrome da imobilidade em pessoas idosas.

\section{MATERIAL E MÉTODO}

É um estudo de revisão de literatura, fundamentada em livros e ensaios científicos publicados sobre a intervenção da fisioterapia na síndrome da imobilidade em idosos.

Para que o artigo fosse construído, trilhou-se um percurso metodológico subdividido em fases. A primeira fase refere-se à formulação da questão norteadora da pesquisa: "Quais as atuais tendências na produção científica nacional e internacional sobre a intervenção fisioterapêutica na síndrome da imobilidade em pessoas idosas abordadas em publicações disseminadas em livros e em periódicos on line na área da Saúde, no período de 2006 a 2016?”. Diante do questionamento elaborado, deu-se seguimento à segunda fase: seleção da literatura direcionada à temática.

Para identificação dos estudos, foi realizado uma busca on line nas bases de dados da Scientific Eletronic Library Online (ScieLO) e da Biblioteca Virtual de Saúde (BVS), onde estão incluso as bases MedLine, e Lilacs e acervos de bibliotecas. Para a localização dos artigos e de outras fontes literárias, foi utilizado como palavras-chaves: Idoso, Imobilidade, Síndrome e Fisioterapia.

Durante a segunda fase foram estabelecidos os critérios de inclusão de estudos, de modo a orientar a busca em banco de dados descritos: estar disponível em livros ou capítulos de livros que abordem a temática; sido publicado na modalidade de artigo científico no formato de ensaio clínico; estar disponível na íntegra, nos acervos de bibliotecas, bem como nas bases eletrônicas já citadas; ter sido publicado entre os anos de 2006 a 2016; estar nos idiomas português, inglês ou espanhol.

No total, foi possível identificar 420 publicações a partir dos descritores selecionados. Após a aplicação dos critérios de inclusão, apenas $\mathbf{7}$ artigos se adequaram à proposta dessa pesquisa. Em seguida, foi elaborado um instrumento de coleta de dados, preenchido para cada artigo analisado, contendo os seguintes elementos: título, autores, ano de publicação, base de dados, fonte, tipo de pesquisa, abordagem e principais resultados. Os dados evidenciados na análise foram debatidos a luz da literatura e apresentados na forma descritiva, a fim de possibilitar a utilidade dessa revisão.

A Figura 1 mostra o fluxograma de busca e rastreio das publicações para análise no presente estudo.

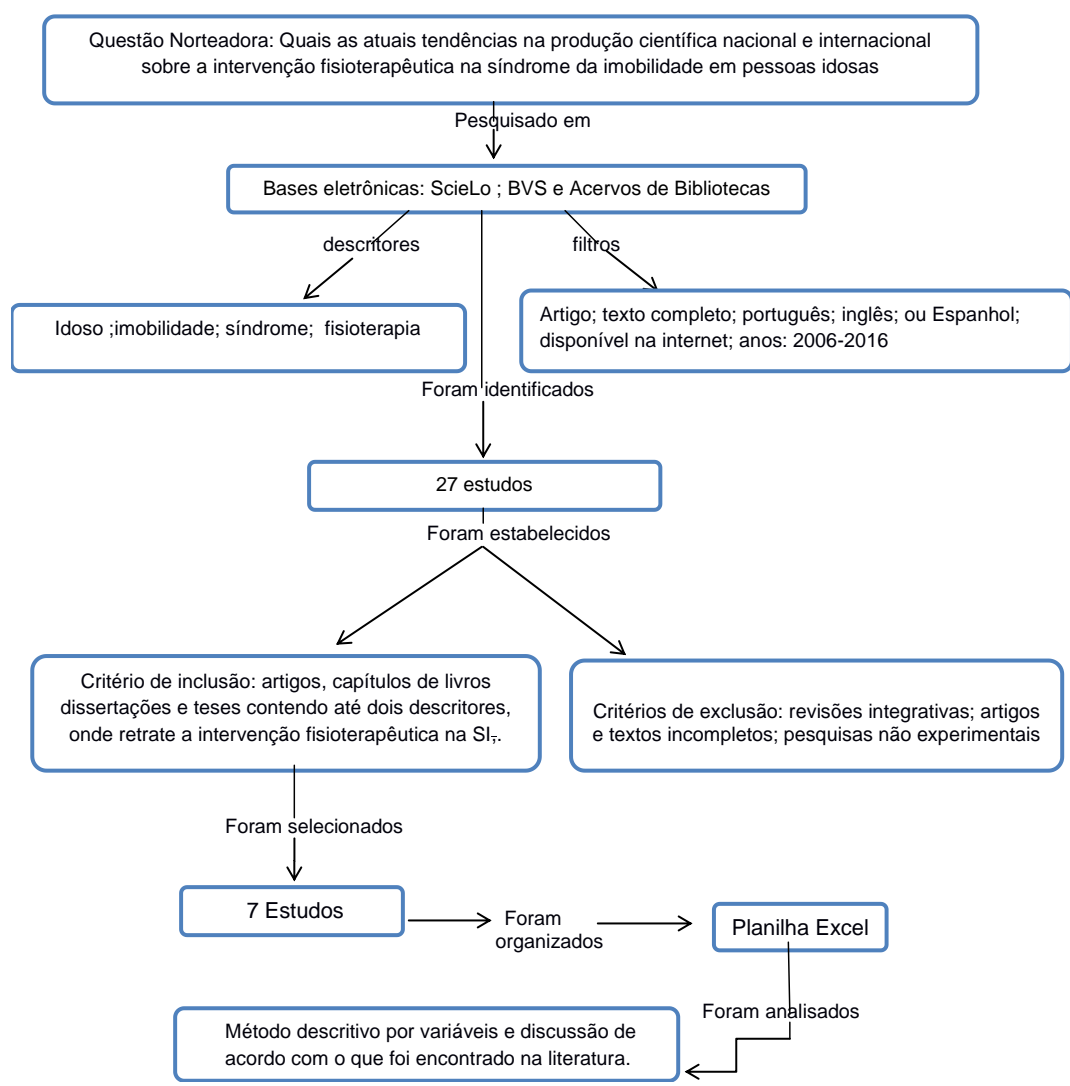

Figura 1: Mapa conceitual do método (Fonte: Dados da Pesquisa, 2017)

\section{RESULTADOS E DISCUSSÃO}

Foram identificados 27 artigos, 1 capítulo de livro, 2 dissertações de mestrado a partir dos descritores selecionados e com utilização dos critérios de exclusão, foram eliminados 23 artigos. Desse modo, a amostra contou com 07 estudos agrupados para análise que cumpriram todos os critérios estabelecidos previamente. Foram construídos quadros visando maior visibilidade dos resultados.

Foi possível observar que, durante os anos de 2010 a 2013, houve um aumento significativo de publicações 
acerca da atuação fisioterapêutica na Síndrome da Imobilidade, tendo como resultados 3 artigos e 1 tese de mestrado, com ápice no ano de 2012. Para Rivoredo e Mejia $^{8}$ a Síndrome da Imobilidade prolongada é um conjunto de disfunções que o corpo sofre, decorrente de um longo período acamado, tendo como seus efeitos, redução na capacidade funcional dos sistemas do corpo e as técnicas mais utilizadas no tratamento da síndrome são a cinesioterapia.

Quadro 1. Relação de artigos para análise por título, autores, ano, fonte e tipo de estudo

\begin{tabular}{|c|c|c|c|c|}
\hline TÍTULO & AUTOR & ANO & FONTE & $\begin{array}{l}\text { TIPO DE } \\
\text { ESTUDO }\end{array}$ \\
\hline $\begin{array}{l}\text { Imobilidade e } \\
\text { síndrome da } \\
\text { imobilização }\end{array}$ & Leduc $^{7}$ & 2006 & $\begin{array}{c}\text { Liv. Tratado } \\
\text { de Geriatria e } \\
\text { Gerontologia. } \\
\text { Cap. } 96 \\
\end{array}$ & Capítulo de livro \\
\hline $\begin{array}{c}\text { Rehabilitácion } \\
\text { funcional del ãnciano }\end{array}$ & Díaz $^{8}$ & 2009 & $\begin{array}{l}\text { Base } \\
\text { eletrônica } \\
\text { Scielo }\end{array}$ & $\begin{array}{c}\text { Revisão } \\
\text { Bibliográfica }\end{array}$ \\
\hline $\begin{array}{c}\text { Atuação } \\
\text { fisioterapêutica em } \\
\text { imobilismo no leito } \\
\text { prolongado }\end{array}$ & $\begin{array}{c}\text { Fernandes e } \\
\text { Leite }^{9}\end{array}$ & 2011 & $\begin{array}{l}\text { Revista } \\
\text { Intellectus }\end{array}$ & $\begin{array}{l}\text { Revisão } \\
\text { Bibliográfica }\end{array}$ \\
\hline $\begin{array}{c}\text { A síndrome do } \\
\text { imobilismo e seus } \\
\text { efeitos sobre o } \\
\text { aparelho locomotor } \\
\text { do idoso } \\
\end{array}$ & $\begin{array}{l}\text { Boechat e } \\
\text { Manhães }^{5}\end{array}$ & 2012 & $\begin{array}{c}\text { Revista } \\
\text { Cientifica } \\
\text { Internacional }\end{array}$ & $\begin{array}{l}\text { Pesquisa } \\
\text { Bibliográfica } \\
\text { Exploratória }\end{array}$ \\
\hline $\begin{array}{c}\text { A cinesioterapia } \\
\text { motora como } \\
\text { prevenção da } \\
\text { síndrome da } \\
\text { imobilidade } \\
\text { prolongada em } \\
\text { pacientes internados } \\
\text { em unidade de terapia } \\
\text { intensiva } \\
\end{array}$ & $\begin{array}{l}\text { Rivoredo e } \\
\text { Meija }^{10}\end{array}$ & 2012 & $\begin{array}{l}\text { Base de dados } \\
\text { eletrônica } \\
\text { Scielo }\end{array}$ & $\begin{array}{c}\text { Revisão } \\
\text { Bibliográfica da } \\
\text { literatura } \\
\text { nacional }\end{array}$ \\
\hline $\begin{array}{c}\text { Síndrome da } \\
\text { imobilidade no idoso }\end{array}$ & Quintela $^{11}$ & 2014 & $\begin{array}{l}\text { Base de dados } \\
\text { eletrônica } \\
\text { Lilacs }\end{array}$ & $\begin{array}{l}\text { Estudo de } \\
\text { Revisão } \\
\text { Sistemática }\end{array}$ \\
\hline $\begin{array}{c}\text { Efeito de um } \\
\text { programa de } \\
\text { mobilização e } \\
\text { exercício ativo sobre } \\
\text { a amplitude articular } \\
\text { em pessoas com } \\
\text { síndrome de desuso }\end{array}$ & Fernandes $^{12}$ & 2015 & $\begin{array}{l}\text { Banco de } \\
\text { Teses e } \\
\text { dissertações } \\
\text { da USP }\end{array}$ & $\begin{array}{c}\text { Estudo } \\
\text { Experimental }\end{array}$ \\
\hline
\end{tabular}

Fonte: Dados da Pesquisa, 2017

Corroborando com o autor supracitado, Boechat e Manhães ${ }^{5}$ descreveram que a Síndrome da Imobilidade como um conjunto de alterações que ocorrem no indivíduo por um tempo prolongado, independente da condição inicial que os motivou ao decúbito prolongado, o repouso beneficia a região lesada, porém o seu prolongamento prejudica o resto do organismo, podendo evoluir para problemas circulatórios, dermatológicos, respiratórios e muitas vezes psicológicos.

Vale ressaltar que nos últimos anos só foi possível encontrar duas publicações de teses como bem demonstra o Gráfico 1. No que diz respeito às modalidades literárias mais vistas, foi possível destacar como principal encontrada a revisão do tipo literária, totalizando quatro (Gráfico 2). No entanto sobre os materiais encontrados de outra categoria foi identificado apenas 1 de cada modalidade literária, sendo eles: livro, revisão sistemática e estudo experimental.

Os resultados e conclusões dos estudos analisados sobre o tema abordado estão apresentados no Quadro 2.

A Síndrome da Imobilidade, apesar de muito descrever um termo muito utilizado pelos geriatras, ainda é pouco conhecida e entendida por outros médicos especialistas, sendo também pouco encontrado com essa nomenclatura, de acordo com Leduc ${ }^{7}$. Sabe-se que é uma síndrome frequente e incidente entre os idosos que geralmente vivem em instituição de longa permanência do idoso (ILPI), que estão acamados em hospitais ou comunidades. Entretanto, o autor referenciado não ressaltou em nenhum momento do seu capítulo formas de tratamento.

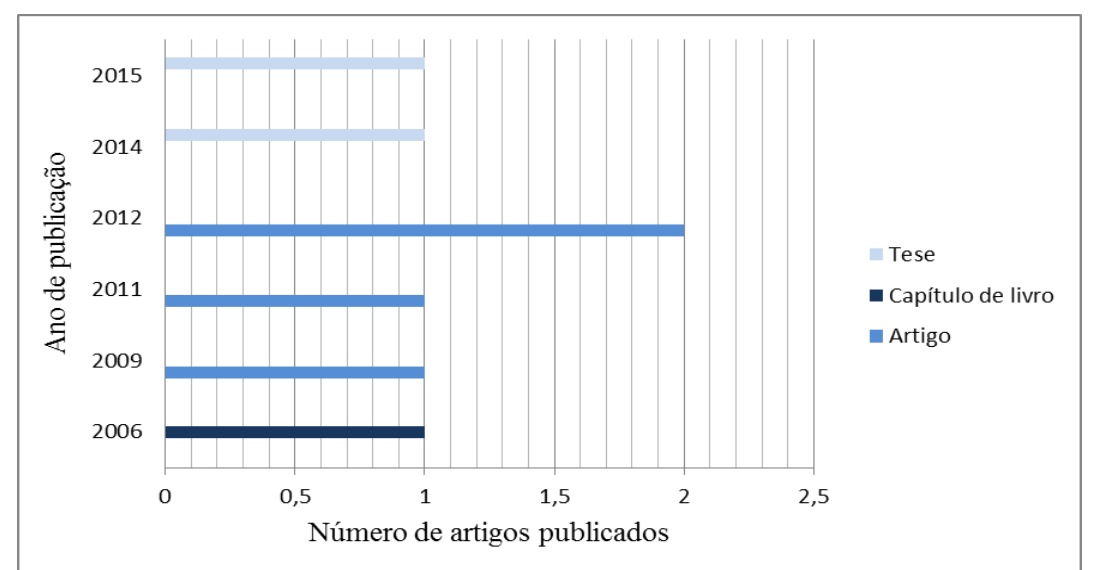

Gráfico 1: Distribuição do número de artigos por ano de publicação (Fonte: Dados da pesquisa, 2017).

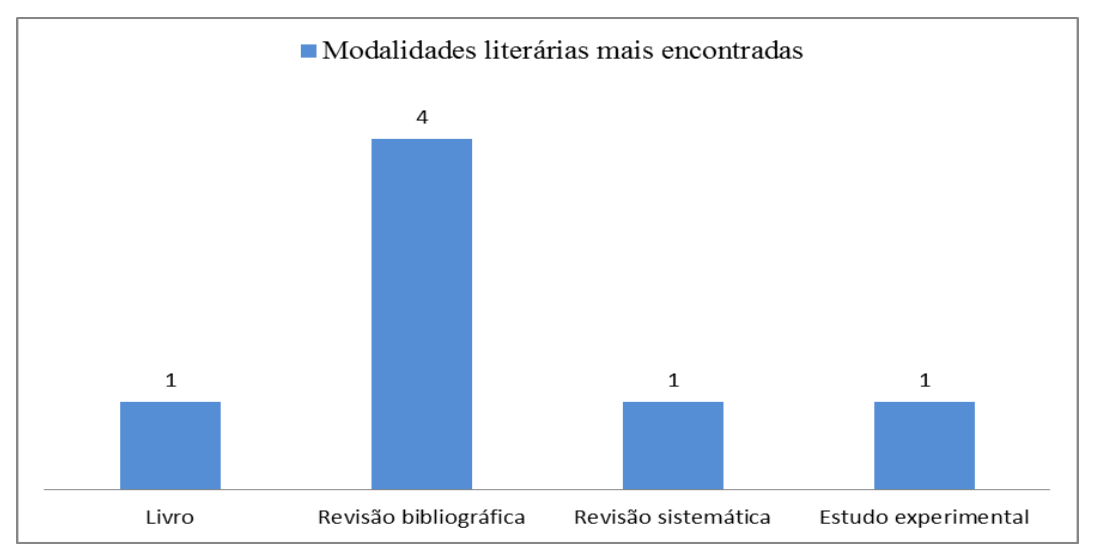

Gráfico 2: Tipos de produções científicas entre os anos de 2006 a 2016 (Fonte: Dados da pesquisa, 2017).

Boechat e Manhães ${ }^{5}$ corroboram as afirmações de Leduc $^{7}$ descrevendo que embora o repouso beneficie o local lesionado, o seu maior prolongamento afeta os demais sistemas, sendo essas complicações aumentadas dependendo dos fatores já existentes de cada pessoa. Para os autores o posicionamento adequado no leito associado a um programa de cinesioterapia é de extrema importância para a prevenção de contraturas osteomusculares e articulares.

Fernandes e Leite ${ }^{9}$ e Quintela ${ }^{11}$ citam ainda que a imobilidade também pode alterar o estado emocional do idoso, independente da condição que levou ao decúbito prolongado, podendo desenvolver um quadro de ansiedade, apatia, depressão, labilidade emocional, isolamento social entre outros. Enfatiza em seu estudo que a fisioterapia tem atuação indispensável no tratamento desses pacientes, minimizando e/ou prevenindo os efeitos deletérios causados pelo prolongado tempo em decúbito.

Rivoredo e Meija ${ }^{10}$ e Rodriguez Díaz ${ }^{8}$ mostram resposta positiva na recuperação dos pacientes com síndrome da imobilidade, sendo possível promover amplitude por menor que seja, corrigir a ineficiência dos músculos e articulações, além de reduzir a perda da capacidade funcional.

Fernandes ${ }^{12}$ em seu estudo sobre síndrome do desuso destacou também que a cinesioterapia, seja ela ativa, passiva ou ativo-assistida, permitiu ganho significativo de amplitude de movimento nas articulações das pessoas com a síndrome. Para tanto o autor usou para a mensuração da amplitude um goniômetro, demonstrando esse diferencial quando comparado aos demais estudos.

A análise dos artigos selecionados mostra que os autores tiveram certa dificuldade na busca de materiais sobre tratamento para a síndrome da imobilidade e relataram escassez em buscar artigos que enfatizassem a atuação fisioterapêutica na síndrome da imobilidade. 
Quadro 2 - Relação de artigos para análise por título, resultados e conclusões.

\begin{tabular}{|c|c|c|}
\hline Artigos & Resultados & Conclusões \\
\hline $\begin{array}{l}\text { A cinesioterapia motora } \\
\text { como prevenção da } \\
\text { síndrome da imobilidade } \\
\text { prolongada em pacientes } \\
\text { internados em unidade de } \\
\text { terapia intensiva }^{10}\end{array}$ & $\begin{array}{c}\text { Os resultados } \\
\text { obtidos atingiram as } \\
\text { expetativas } \\
\text { propostas pelo } \\
\text { estudo. }\end{array}$ & $\begin{array}{c}\text { Foi possível observar que é de } \\
\text { extrema importância à } \\
\text { atuação do fisioterapeuta } \\
\text { nesse âmbito, uma vez que } \\
\text { teve o atendimento } \\
\text { diferenciado visando uma } \\
\text { evolução satisfatória. }\end{array}$ \\
\hline $\begin{array}{c}\text { A síndrome do } \\
\text { imobilismo e seus efeitos } \\
\text { sobre o aparelho } \\
\text { locomotor do idoso }{ }^{5}\end{array}$ & $\begin{array}{l}\text { Observou-se que os } \\
\text { efeitos da } \\
\text { imobilização no } \\
\text { organismo vão além } \\
\text { de contraturas, } \\
\text { podendo resultar } \\
\text { efeitos deletérios no } \\
\text { corpo. }\end{array}$ & $\begin{array}{l}\text { Este estudo permitiu entender } \\
\text { que quanto mais precoce a } \\
\text { fisioterapia em pacientes } \\
\text { imobilizados, menores serão } \\
\text { os efeitos danosos no corpo } \\
\text { deles. }\end{array}$ \\
\hline $\begin{array}{l}\text { Atuação fisioterapêutica } \\
\text { em imobilismo no leito } \\
\text { prolongado }\end{array}$ & $\begin{array}{c}\text { Os danos } \\
\text { decorrentes da } \\
\text { imobilização } \\
\text { prolongada foram: a } \\
\text { diminuição da força } \\
\text { muscular, } \\
\text { incapacidade } \\
\text { funcional geral, e a } \\
\text { falta de instrução as } \\
\text { famílias, cuidadores } \\
\text { e profissionais. }\end{array}$ & $\begin{array}{c}\text { A confecção de um manual é } \\
\text { de extrema importância nesse } \\
\text { caso no intuito de contribuir } \\
\text { com efetividade o tratamento } \\
\text { fisioterapêutico e } \\
\text { consequentemente resultados } \\
\text { positivos. }\end{array}$ \\
\hline $\begin{array}{l}\text { Efeito de um programa de } \\
\text { mobilização e exercício } \\
\text { ativo sobre a amplitude } \\
\text { articular em pessoas com } \\
\text { síndrome de desuso }{ }^{12}\end{array}$ & $\begin{array}{l}\text { Foram verificados } \\
\text { resultados } \\
\text { estatisticamente } \\
\text { significativos nas } \\
\text { articulações dos } \\
\text { MMII após a } \\
\text { aplicação do } \\
\text { exercício de } \\
\text { mobilização e } \\
\text { exercício ativo. } \\
\end{array}$ & $\begin{array}{l}\text { Foi visto no estudo que um } \\
\text { programa de mobilizações } \\
\text { articulares, implementado } \\
\text { com regularidade, pode } \\
\text { contribuir para a melhoria das } \\
\text { amplitudes articulares em } \\
\text { pessoas com síndrome do } \\
\text { desuso(imobilidade) }\end{array}$ \\
\hline $\begin{array}{l}\text { Imobilização e síndrome } \\
\text { da imobilização }\end{array}$ & $\begin{array}{c}\text { Ocorre à diminuição } \\
\text { de todos os sistemas } \\
\text { do corpo humano, } \\
\text { seja ele do mais } \\
\text { simples ao mais } \\
\text { complexo, trazendo } \\
\text { com isso um quadro } \\
\text { irreversível da } \\
\text { doença quando o } \\
\text { idoso encontra-se } \\
\text { num quadro } \\
\text { avançado. }\end{array}$ & $\begin{array}{c}\text { A síndrome da imobilidade } \\
\text { em um estado avançado é } \\
\text { grande sofrimento para o } \\
\text { paciente e seus familiares. É a } \\
\text { fase em que a degradação da } \\
\text { qualidade de vida chega ao } \\
\text { limite do tolerável ou } \\
\text { aceitável. É um caminho sem } \\
\text { volta, e, para o paciente, a } \\
\text { morte acaba sendo a melhor } \\
\text { solução diante dos } \\
\text { sofrimentos, pode-se dizer } \\
\text { que a vida está sendo } \\
\text { representada apenas pela } \\
\text { matéria. }\end{array}$ \\
\hline $\begin{array}{c}\text { Rehabilitácion funcional } \\
\text { del ãnciano }\end{array}$ & $\begin{array}{c}\text { Existem fatores } \\
\text { intrínsecos e } \\
\text { extrínsecos para as } \\
\text { causas de } \\
\text { dependência nos } \\
\text { idosos, no entanto já } \\
\text { existe tratamento } \\
\text { fisioterapêutico, } \\
\text { para que haja a } \\
\text { diminuição desses } \\
\text { fatores agravantes. }\end{array}$ & $\begin{array}{l}\text { Ainda há muito a ser feito no } \\
\text { campo da reabilitação para } \\
\text { conseguir a satisfação e } \\
\text { qualidade de vida que os } \\
\text { idosos precisam, no entanto } \\
\text { não foram encontradas } \\
\text { barreiras ou obstáculos à } \\
\text { participação ativa dos idosos } \\
\text { em atividades sociais. }\end{array}$ \\
\hline $\begin{array}{l}\text { Síndrome da imobilidade } \\
\text { no idoso }{ }^{11}\end{array}$ & $\begin{array}{l}\text { A avaliação da uma } \\
\text { melhora nos índices } \\
\text { de dependência por } \\
\text { ser uma ferramenta } \\
\text { de extrema } \\
\text { importância, } \\
\text { possibilitando } \\
\text { identificar casos } \\
\text { precoces do quadro } \\
\text { de imobilismo. }\end{array}$ & $\begin{array}{l}\text { A identificação de padrões } \\
\text { clínicos e analíticos mais } \\
\text { precisos possibilitaria a } \\
\text { compreensão mais profunda } \\
\text { dos efeitos deletérios da } \\
\text { imobilidade. O presente } \\
\text { estudo propõe a realização de } \\
\text { mais estudos na área. }\end{array}$ \\
\hline
\end{tabular}

\section{CONCLUSÃO}

Baseando-se na amostra dos dados, foi possível observar que a atuação fisioterapêutica na Síndrome da Imobilidade é de extrema eficácia na prevenção dos agravos antes e após a sua instalação. Os artigos analisados destacam a cinesioterapia como recurso fisioterapêutico mais frequente e com resultados impactantes nas repercussões negativas que a doença traz. Entretanto, observou-se que muito ainda se tem de investigar sobre esta temática.

Após esse estudo fica como sugestão a construção de mais artigos na área da fisioterapia relatando os benefícios que ela traz nos agravos da Síndrome da Imobilidade, através de publicações de estudos práticos e principalmente escritos por profissionais de fisioterapia.

\section{REFERÊNCIAS}

1. Costa, EFA, Pereira, SRM. Meu corpo está mudando o que fazer? Tempo rio que arrebata (p.13-25),2005. In: Pacheco JL, Sá JLM, Py L, Goldman SN (orgs.). Tempo rio que arrebata. Holambra: Setembro; p.13-25

2. Cazeiro, APM, Peres, PTA Terapia cupacional na prevenção e no tratamento de complicações decorrentes da imobilização no leito. Cad Bras Ter Ocup. 2010; 18(2):149-67.

3. Neri AL. Palavras-chave em gerontologia. Campinas: Alínea; 2014

4. Moraes EM (org). Princípios básicos de geriatria e gerontologia. Belo Horizonte: Coopemed; 2008.

5. Boechat JCS, Manhães FC, Gama Filho RV, Istoé RSC. A síndrome do imobilismo e seus efeitos sobre o aparelho locomotor do idoso. InterScience Place. 2012; 1(22):89-107.

6. Melo ACR, López RFA. Efeitos da atividade física na redução dos efeitos do imobilismo Revista Digital. Buenos Aires, ano 10, n. 68, Janeiro de 2004.

7. Leduc MMS. Imobilização e síndrome da imobilização. In: Freitas EV, Py L (orgs). Tratado de geriatria e gerontologia. 2.ed. Rio de Janeiro: Guanabara Koogan; 2006

8. Rodríguez Diaz O. Rehabilitación funcional del anciano, MEDISAN. 2009; 13(5):2009

9. Fernandes F, Leite J, Nascimento B, Baciuk EP. Atuação fisioterapêutica em imobilismo no leito prolongado. Revista Intellectus. 2011; 9(25).

10. Rivoredo MGAC, Meija D. A Cinesioterapia Motora como prevenção da Síndrome da Imobilidade Prolongada em pacientes internados em Unidade de Terapia Intensiva. Disponível em: http://portalbiocursos.com.br/ ohs/data/docs/27/15__A_Cinesioterapia_Motora_como_ prevenYYo_da_SYndrome_da_Imobilidade_Prolongad a_em_pacientes_internados_em_UTI.pdf. Acesso em: 16 mai. 2016.

11. Quintela JMRF, Síndrome da imobilidade no idoso [dissertação]. Coimbra: Universidade de Coimbra, Portugal; 2015.

12. Fernandes TJ. Efeito de um programa de mobilização e exercício ativo sobre a amplitude articular em pessoas com síndrome de desuso [dissertação]. Bragança:Instituto Politécnico de Bragança, Escola Superior de Saúde, Portugal; 2015.

\section{CONFLITO DE INTERESSES}

Os autores declaram não haver conflitos de interesse.

\section{AUTOR PARA CORRESPONDÊNCIA}

Hellen Cristina Barbosa Pereira

hellen.c.barbosa@hotmail.com

Submetido em 23/08/2017 Aceito em 28/09/2017 